\title{
Rencontre avec Daniel Mesguich, traducteur et metteur en scène de Titus Andronicus
}

Jean-Michel Déprats

\section{(2) OpenEdition}

\section{Journals}

Édition électronique

URL : http://journals.openedition.org/shakespeare/1204

DOI : 10.4000/shakespeare. 1204

ISSN : 2271-6424

Éditeur

Société Française Shakespeare

Édition imprimée

Date de publication : 1 novembre 1991

Pagination : 189-203

Référence électronique

Jean-Michel Déprats, «Rencontre avec Daniel Mesguich, traducteur et metteur en scène de Titus Andronicus », Actes des congrès de la Société française Shakespeare [En ligne], 9 | 1991, mis en ligne le 01 janvier 2007, consulté le 01 mai 2019. URL : http://journals.openedition.org/shakespeare/1204 ; DOI : 10.4000/shakespeare.1204 


\section{SHAKESPEARE \\ ET LE CORPS A LA RENAISSANCE}

Société Française Shakespeare

Actes du Congrès 1990

sous la direction

de

M.T. JONES-DAVIES

PARIS

LES BELLES LETTRES

1991 
Tous droits de traduction, de reproduction et d'adaptation réservés pour tous les pays.

(C) 1991 Société d'édition Les Belles Lettres, 95 bd Raspail 75006 Paris

ISBN $2.25169117-\mathrm{X}$ 


\title{
Rencontre avec Daniel Mesguich traducteur et metteur en scène de Titus Andronicus
}

\author{
animée par Jean-Michel Déprats
}

JMD : Nous remercions Daniel Mesguich, vieux compagnon de la Société Shakespeare, que nous avons toujours plaisir à accueillir et qui soustrait un temps précieux à des répétitions pour venir nous parler de sa mise en scène de Titus Andronicus donnée au théâtre de l'Athénée en novembre 1989. Il a apporté des photos comme points d'appui puisque le spectacle était visuellement très beau. Des tournées de ce spectacle sont prévues en octobre, novembre, décembre 1990 dans toute la France.

Daniel Mesguich a des rendez-vous fréquents avec Shakespeare : en 1977 le premier Hamlet créé à Grenoble, repris au Théâtre des Amandiers de Nanterre, Le Roi Lear en 1981 au Palais des Papes d'Avignon, Roméo et Juliette en 1985, un nouvel Hamlet dans lequel Daniel Mesguich jouait le role-titre en 1986 au théâtre Gérard Philippe de Saint-Denis. C'est donc sa quatrième mise en scène shakespearienne. Avec Titus Andronicus, on est entré dans une autre période. Durant longtemps, la paternité en était contestée. Cette pièce est d'actualité sur les scènes: Peter Brook, en 1955, a très largement contribué à une redécouverte de la pièce dans une mise en scène dont les principaux acteurs étaient Laurence Olivier et Vivian Leigh. Depuis lors, on la joue très régulièrement en Angleterre, beaucoup plus fréquemment que d'autres pièces comme Pericles ou Cymberline. Même en France l'actualité théâtrale l'a souvent remise à l'affiche : en dix ans on ne compte pas moins de quatre mises en scène : une de Beuglin au Festival d'Avignon avec Bruce Meyers, une au Théâtre de la Villette avec Pierre Bilan, une plus récente de Michel Dubois au Théâtre de Chaillot et celle-ci, aussi différente des trois autres que possible. Cette fréquence ne diminue pas les difficultés d'approche car l'un des problèmes au théâtre est comment représenter cette série d'atrocités qui font la notoriété de la pièce. Michel Dubois avait choisi le parti-pris d'un grand guignol au sens thêâtral qui flirtait 
avec l'univers de Beneix, mais avec des réussites intermittentes: le grand guignol vire très vite au guignol, parfois ce virage est voulu, parfois il se fait au détriment du spectacle, des acteurs, à travers une option qui n'est pas délibérée. Pour être complet, sur la scène française, bien que ce soit une troupe anglaise, on a pu voir en mai-juin dernier, la mise en scène de Deborah Warner avec Brian Cox qui viendra nous parler de Titus. Il faut mentionner un Titus Andronicus mis en scène par Peter Stein en Italie qui va venir au Théâtre de l'Europe à la fin de la saison et une autre, assez récente, de Matthias Langhoff, en Allemagne. Nous allons commencer par parler du texte : une nouveauté, c'est toi qui le signe. Après une collaboration avec Michel Vitez pour Hamlet dont on avait parlé longuement car ce texte tentait d'être l'équivalent fictif d'un écrit français du XVIe siècle d'un auteur imaginaire Guillaume de Branlans. Ce texte essayait de travailler dans l'archaïsme, en tension avec des modernités, à mi-chemin entre Maurice Scève et Lacan. Tu avais demandé à Gervais Robin, comédien et metteur en scène, de faire l'adaptation de Roméo et Juliette. Ton texte n'est pas une traduction au sens strict, c'est un montage. La trame verbale reprend celle de François-Victor Hugo, ce qui n'est nullement une remarque critique. Il y a aussi un montage textuel. On pourra parler des coupures, de leur nature et de leur fonction et des adjonctions qui orientent le sens.

DM : II ne s'agit pas d'une adaptation de la traduction de François-Victor Hugo. Adaptation certes, mais à partir du texte anglais de Shakespeare, en m'aidant de la traduction la plus répandue qu'on trouve dans les collections de poche et de deux ou trois autres auteurs dont une collection bilingue avec douze pieds par vers, et j'ai pris des dictionnaires. On pourrait décrire ma démarche en trois grands pans. Le premier, minoritaire, en m'aidant de la traduction de $X, Y$, ou $Z$, trouvant la formule intéressante même un peu loin du texte littéral. Ensuite je suis mon envie de le rendre plus lyrique ou plus fort en langue française. Il s'agit d'un travail de traduction au sens large. Troisièmement: je pars d'un principe, peut-être contestable quant à la littéralité, mais qui me parait juste par rapport à Shakespeare. Shakespeare est un véritable continent à lui tout seul. Bien qu'il s'agisse d'un homme, il a écrit toutes ces pièces l'une après l'autre, pas comme un ordinateur devenu fou qui fait tout gicler en même temps. Cela me paraît important de savoir que celui qui a écrit Othello a aussi écrit $L e$ 
Roi Lear, Titus Andronicus, Macbeth, etc. Une lecture sourde et aveugle quant aux autres pièces, toute intelligente soit-elle, serait fausse. Il y a des connections obligatoires à faire, ne serait-ce que parce qu'on retrouve certains personnages, certaines structures, certains canevas, certains rappels et parfois certains vers quasiment intégralement. J'avais évoqué une image à propos de Roméo et Juliette. Dans certains greniers à humidité très forte, au bout de quarante ou cinquante ans, le papier d'un très beau livre se trouve imbibé d'eau, et du coup, on peut lire en transparence la page qui est derrière. C'est comme si Le Roi Lear ou Macbeth avait légèrement imbibé Titus Andronicus. Lorsqu'il y a une tirade de Titus Andronicus qui, à mon avis, était moyenne, même si tel ou tel vers sortait comme une petite merveille au milieu de quelque chose d'assez mou théâtralement et pour nous aujourd'hui, il y a à peu près la même dans Le Roi Lear sur cinq ou six vers et c'est génial. Je n'avais qu'à prendre ces vers et les glisser dans Titus Andronicus, ni vu ni connu et Titus Andronicus s'en trouvait enrichi : on ne peut pas ne pas entendre Tamora comme l'écho assourdi de Lady Macbeth, par exemple; les deux fils comme Iago ou bien les assassins dans Richard III. Aaron le Maure comme Iago ou Edmund le bâtard dans Le Roi Lear ou Titus Andronicus comme Le Roi Lear lui-même, ou Capulet père. Au bout d'un moment, je me suis mis à traduire et pourtant à adapter largement, mais chaque fois à partir de l'anglais, même les petits bouts du Roi Lear ou de Macbeth. J'ai donc eu une pièce protéiforme, mais avec la structure à peu près exacte de la pièce de Shakespeare, en tous cas les thèmes qui me paraissaient essentiels étaient préservés. Ce que les acteurs avaient en bouche était à mon sens plus beau qu'une traduction littérale, et comme on fait avant tout du théâtre, c'est-à-dire qu'il ne s'agit pas de laisser une œuvre écrite, je me suis dit que tout allait bien et que l'on jouait effectivement Titus Andronicus.

JMD : Ma remarque n'était nụllement professorale. Heiner Müller, par exemple, fait aussi un travail de ré-écriture en ayant le texte anglais comme référence. Il est intéressant de remarquer différents rapports au texte. Même ce type d'intervention sur la traduction de François-Victor Hugo est une démarche habituelle au théâtre qui n'est pas à récuser en soi. La traduction de F.-V. Hugo a certainement un mérite incontestable : son honnêteté, son exactitude, et un démérite non moins évident aujourd'hui, car le rythme d'ensemble n'est pas très porteur de gestes. Le simple fait 
de pratiquer des coupures, de faire ressortir la trame narrative ou la trame de l'imaginaire, c'est donner naissance à un texte autre, plus fort parfois, quand il y a cette perle dans la gangue d'une coulée verbale qui n'est pas toujours très rythmée. Les adjonctions sont toujours significatives, un petit exemple mais qui en dit long sur le pourquoi et le comment de la pièce. Dans la pièce de Shakespeare la demière tirade est à Lucius, dans le texte que tu signes, la dernière réplique est attribuée à Marcus Andronicus : «Comment une telle horreur a-t-elle été possible? Qui fit pénétrer la fatale machine qui porta le désordre dans la cité?» Le dernier mot n'est pas choisi au hasard par l'auteur ou l'adaptateur.

DM : Ceci nous mène très loin. Je vais vous exposer les principales lignes qui ont présidé à l'établissement de cette mise en scène avant d'expliquer pourquoi Marcus termine ainsi. La raison pour laquelle j'ai voulu monter Titus Andronicus est presque inavouable. Au milieu de ce ramollissement général des formes, du théâtre, du cinéma et même de la littérature, quand s'énerve-t-on un peu? Dans sa jeunesse, il avait trente ans, Shakespeare avait écrit une pièce d'une violence incroyable. Cette première raison un peu bébête est idéologiquement obéissante : le bruit courant que Titus Andronicus était une pièce d'horreur, de sang, de meurtre, de mutilations, de viol, de langues et de mains arrachées, je me suis dit que cela nous réveillerait peut-être. Deuxièmement, en lisant le texte activement en vue de le travailler, je me suis rendu compte que le sang, l'hémoglobine, n'était pas le plus important. J'ai trouvé cette pièce diffamée. On dit toujours que Titus Andronicus est une pièce de violence, bien sûr on coupe les mains et la langue de Lavinia, mais d'abord, cela peut se lire comme un symbole : la langue pour parler, la main pour écrire. C'est du sang symbolique, comme couper la tête des deux fils. Shakespeare est toujours dans le champ symbolique. Il a écrit cette pièce après une épidémie de peste, parce que le Globe avait brûlé, il avait tourné dans les campagnes et a présenté une pièce très violente devant un auditoire paysan qui n'aurait pas compris les subtilités. Je crois que cette pièce est majeure, c'est la première tragédie que Shakespeare ait écrite, celle qui serait l'assise de toutes les autres. Cette pièce raconte très exactement les problèmes et les horreurs qui s'ensuivent quand on n'a pas assez fait confiance au symbolique. Comment commence la pièce? Un général revient à Rome après sa campagne contre les Goths, les barbares. Comme souvent chez Shake- 
speare, c'est un bouleversement, une dualité troublante et mouvante. Le vieil empereur vient de mourir, il s'agit de choisir un empereur, les deux fils se divisent pour le pouvoir. Ce vieux général qui a fini sa vie, qui rentre couvert d'honneurs et qui devrait regarder tranquillement la télévision le soir, est pressenti pour être empereur. Sa première réaction est de refuser, on se dit : voilà un homme intègre. Mais on s'aperçoit qu'il a dit non mais continue quand même à diriger Rome, et c'est lui qui nomme l'empereur. Il se met su-dessus de l'empereur, pour les mannes de ses fils morts au combat - il en a vingt-cinq en tout, un peu comme dans la Bible. On est dans du symbolique, du mythologique, de l'allégorique, du légendaire, à ne pas prendre au pied de la lettre. On ne parle pas de Rome, ce n'est pas une dramatique historique d'Alain Decaux, mais du mental, de quelque chose de plus fort. Il s'agit de faire un sacrifice, comme on en a l'habitude. Son fils vivant, rescapé, Lucius, celui qui va devenir empereur évidemment, dit : si au lieu de faire comme d'habitude on tuait le fils vivant de la reine Tamora, une barbare prisonnière ramenée à Rome, ce serait encore mieux. Comme si on n'avait pas eu assez confiance dans ces rituels habituels, dans le symbole normal. Si on saupoudrait tout cela de sang, ce serait encore meilleur. Voilà le vrai thème de Titus Andronicus. Le symbole ne suffit pas, il va falloir l' achever, aller plus loin encore, l'incarner - au sens littéral - mettre de la chair dedans, broyer de la chair. On peut penser à Kafka, à La Colonie Pénitentiaire, à Beyrouth, au terrorisme actuel, à l'affaire Rushdie. Il y a comme une faillite du symbole - à la place, on met de la chair humaine, pourquoi pas? Le Christ n'a rien fait d'autre : il a dit jusqu'à présent vous aviez le livre, cela ne suffit pas, prenez ma chair et mon sang, c'est encore meilleur. Pour l'un des versants, pour Titus Andronicus et les Romains, c'est plus "hard", plus agréable, mais pour l'autre versant, Tamora, il n'y a pas de symbole qui tienne, c'est son fils qu'on vient de tuer, cela ne se décrypte pas, c'est de l'immédiat, de la chair, du vivant, de l'horreur. Mais voilà où Shakespeare est génial : au bout de dix minutes tout s'inverse parce que Tamora devient impératrice et Titus devient son sujet, et on se dit que la vengeance va aller bon train. Pour qu'il n'y ait plus de mauvais comptes qui restent, on tue un fils de Tamora, et maintenant, ça y est, c'est zéro-zéro, on peut recommencer, les pendules sont à l'heure. Mais Shakespeare nous dit: ne remettez jamais les pendules à l'heure, laissez-les comme elles sont, ne faites pas table rase. Chaque fois que vous faites cela, 
quelqu'un est écrabouillé au passage, et pour ce quelqu'un-là, les pendules ne sont pas du tout à l'heure, ça recommence de plus en plus violemment. C'est une leçon politique, morale, philosophique, sublime. C'est aussi une sorte d'anti-Bible : quel souvenir peut-on avoir d'un sacrifice pour apaiser une volonté quelconque extérieure, transcendante? Le sacrifice d'Abraham, qui lève son couteau devant Isaac son fils. Dieu dit non, ce n'est pas la peine, c'était pour rire, pas de sang, restons kasher. Il ne le tue pas, mais à la place, il met une brebis, et le sacrifice va bien, on est dans le rituel normal. L'Alliance n'a pu se faire que parce qu'il n'y avait pas de sang humain versé. Alors qu'ici, c'est exactement l'inverse qui se passe, et qu'on a joué nous, puisqu'au départ, il y avait une espèce de tête d'animal empaillée, une espèce de squelette, une chose à lire, symbolique et non pas une chose vivante et on tue à la place un homme vivant. Ce processus se retrouve sans arrêt tout au long de la pièce, par exemple, Lavinia qui a la langue et les mains coupées. Mais il y a mille façons de communiquer; pourtant Shakespeare dit qu'on ne peut rien savoir d'elle. C'est un corps vivant, mais elle est comme morte, elle n'a plus de langue, de langage. Elle n'existe plus, et pourtant, son corps continue à errer. Elle est là sans avoir de vrais rapports, de vrai commerce avec les autres. Et pourquoi si tard, au moins six mois après, Lavinia lève les bras au ciel pour montrer un livre qui appartient au jeune Lucius dans la pièce initiale, - nous n'avons pas maintenu ce personnage. Titus se tourne vers Marcus pour avoir une explication. Cette phrase : «Qu'est-ce que ça veut dire?» revient souvent. Les personnages s'arrêtent et disent: «Essayons de lire, de décrypter cela, y a-t-il une solution?» En général, c'est Marcus qui répond. Les deux frères sont complètement différents. L'un a été général, l'autre a vaguement été prêtre défroqué, bibliothécaire, c'est lintello, un tribun, un homme politique, alors que l'autre, est le physique. Il dit : cela peut vouloir dire deux choses : ou bien elle lève les bras au ciel parce qu'elle veut signifier que deux personnes l'ont violée, assassinée en quelque sorte, ou bien tout simplement pour attraper un livre. C'est indécidable. Plus tard, on lui donne un livre. Titus questionne à nouveau, Marcus répond que dans ce livre il y a l'histoire métaphorique de ce qui lui est arrivé, ou bien qu'il appartenait à sa mère qu'elle aimait. Le symbole n'arrive plus bien à se lire; il faut donc décider arbitrairement, littéralement trancher, mais en tranchant, parfois on coupe des bras, des langues. Pourquoi Marcus a-t-il le dernier mot? Parce que 
toute cette pièce s'est passée dans le champ de Marcus. Au theâtre, il n'y a pas que du symbole, mais aussi de la chair humaine, les acteurs. Voilà pourquoi à mon avis, le théâtre est pacifique, démocratique, ouvert, symbolique, alors qu'au contraire, l'affaire Rushdie, s'il avait été tué, ou telle guerre fratricide n'est pas du théâtre parce que le sang coule pour de bon, on ne se relève pas. Marcus termine parce que dans le champ symbolique, il risque d'y avoir des moments de faillite, des moments où on n'a plus assez confiance en nos civilisations, en nos cultures, en nos livres, en nos réflexions, en nos rêves, en nos rituels, en ce qui est cuit, comme dirait Lévy-Strauss, et non pas en ce qui est cru comme le sang humain qui coule. De temps à autre on éprouve le besoin de boucher les trous et les faillites de ce cuit, de cette culture, de ce réchauffé, au sens noble du terme, de tout le champ symbolique, par du vivant, de la chair humaine, du sang, du cru, et en général, c'est l'horreur dit Shakespeare. C'est une bonne leçon de morale.

JMD : Passons au décor, puisque cela vient dans la droite ligne de ce que tu viens de dire, sur le livre symbolique. Il y a souvent des bibliothèques. Dans ce spectacle, une image récurrente emblématise la poésie thêtrale : à chaque fois qu'un crime est commis, un livre s'enflamme. Peut-on décrire rapidement le décor et dire le pourquoi de cette bibliothèque? Un autre élément de décor, sur la droite représente un aquarium sur lequel flotte des corps morts comme des poissons - cela tient du bloc opératoire, de la Bibliothèque Nationale, du Mystère des Jardiniers, avec au centre une verrière-hublot.

DM : Une coupole renversée. C'est une bibliothèque en effet, mais que j'espère infinie, borgésienne, de Babel. Ce sont des gens qui ne savent pas lire, qui n'ont plus confiance en ce qui est écrit. Ils infestent ce lieu, ils se promènent, ils peuvent dormir sur des livres. Un peu comme il peut nous arriver de ne plus trop savoir à quoi servait telle ou telle chose antique. On peut imaginer que quelqu'un tende un fil pour faire sècher du linge entre deux menhirs, on utilise ce qui dépasse, alors qu'il s'agit d'une chose intelligente, empreinte de rituel. Il peut y avoir des incultures, on se ressert des choses autrement. Ces gens-là vivent dans cette bibliothèque en se disant : c'est bien commode des livres, ça permet de monter comme des marches, on peut se coucher dessus, ils sont assez moelleux, surtout quand ils sont pourris, vermoulus. 
Mais ils ne savent plus forcément les lire. C'est une manière un peu violente, rapide, de dire non pas que c'est un moment de décadence au sens moral du terme, mais un moment où le symbolique est en train de perdre de sa force. Jusqu'à Champollion, on pouvait dire que les Egyptiens faisaient des petits dessins sur les murs. On ouvre des livres qu'on peut tenir à l'envers. Titus Andronicus se passe dans une Rome un peu analphabète du passé, plutôt que décadente, ou du Bas-Empire. D'autre part, cette bibliothèque n'est pas droite, elle est couchée, à l'envers : ce qui devrait être en haut est en bas ou plutôt est lointain, comme si vous vous promeniez dans une cour intérieure entre quatre immeubles parisiens, que vous vous allongiez par terre et regardiez le ciel juste droit devant vous. Les choses sont littéralement insensées, elles n'ont plus de sens, où est le haut, le bas, la droite ou la gauche? Puisque toute cette pièce pose la question du sens, on avait intérêt à faire un décor qui essaie d'échapper au sens normal. D'ailleurs le théâtre lui-même vit toujours un peu là-dessus. Il n'y a qu'au théâtre, travail sur la re-lecture des choses en traversant des corps vivants en direct et non pas dans des études, que l'on peut dire à un acteur : là il serait bon que tu remontes vers le fond, c'est à dire aller vers le lointain, mais dites à un scaphandrier de remonter vers le fond!

Le théâtre au départ joue avec le sens liftéral. Les vivaria, ou plutôt les mortaria sur les côtés seraient des espèces de serres avec du vivant, de la nature : on y met les morts, lhumus, la terre. J'avais beaucoup pensé à certains écrits de Michel Serres. C'est là qu'il y a la terre, la mort, mais aussi la vie, c'est là qu'on met les cadavres qui se décomposent et qui sont les premières statues, les premières choses religieuses. C'est comme si un lieu culturel avait été perforé et ouvert, crevé par du naturel. En général, c'est le contraire : la nature que l'on représente est plutôt infinie, on n'en voit pas le bord, dans un théâtre en tous cas. Un lieu culturel serait un lieu fermé. Là, c'est la bibliothèque qui est infinie, morte comme un cercueil; la vie des plantes, des corps arrivent et viennent crever les livres, comme si la végétation qui était dehors était rentrée. C'est ce qui arrive dans tout processus d'entropie banal. Si on laisse un temple même magnifique au milieu de la jungle, au bout de quelques années ou siècles, la végétation finit par entrer. C'est un peu cela qu'on jouait.

Pourquoi un livre en flammes tombe à chaque fois qu'on tue quelqu'un? C'est la proposition inverse de : "un bébé - un arbre"; 
"un corps humain - un livre qui meurt", comme dans certaines civilisations indiennes, la tribu Hopi, je crois, quand quelqu'un meurt, on annule un mot de la langue. Maintenant, cette tribu a un langage extrêmement pauvre. Un livre se consumme et disparaît à jamais. La vie est en quelque sorte dans les livres. On est à michemin entre la vie et la mort. Titus Andronicus se passe dans cet entre-deux banal chez Shakespeare : les remparts de Hamlet, dedans Elseneur, dehors la mer; dans Romeo et Juliette le jour et la nuit, l'alouette et le rossignol. Titus Andronicus est entre le livre et le corps, entre deux règnes aussi : il y a deux empereurs Saturninus, empereur fantoche qui ne fait que préparer Lucius, entre le livre vivant et la liane ou la ronce qui, bien que vivante est la mort.

JMD : Ce n'était pas quelque chose à décrypter en permanence durant le spectacle, il y avait une grande poésie thêâtrale.

DM : C'était beaucoup plus simple que ce que je viens de raconter.

JMD : Le spectacle n'appelait pas une démarche de type intellectualiste. Les significations pouvaient parvenir par le moyen du théâtre, des gestes, des récurrences, des rythmes. Le "montage" textuel, le déroulement du spectacle de type cinématographique, composé par tableaux, qui se closent par la chute du rideau.

DM : On ne coupait pas que les mains et les têtes, mais aussi le texte, le rideau était une sorte de guillotine, comme au cinéma le montage-cut, ou les élipses que sont un plan sur l'autre.

JMD : Cela traduisait le choix de ne pas prendre la pièce dans son déroulement dramatique, mais de montrer l'éternel retour du même, le ressassement de l'histoire, la répétition de l'atrocité.

DM : C'est une suite de variations sur le premier meurtre : le sacrifice du premier fils de la reine Tamora.

JMD : Ce qui explique le choix esthétique de représentation.

DM : Je n'ai pas vu la représentation de Michel Dubois qui paraît-il était très intéressante, ni celle de Peter Brook, qui était symbolique, mais il me semble qu'elles figurent les deux extrêmes. 
JMD : Mais tu as vu celle de Deborah Warner avec Brian Cox, une des meilleures que l'on peut imaginer, qui utilise le grand guignol.

DM: $\quad$ Ce qu'on m'a dit, c'est que celle de Dubois était réaliste dans le grand guignol, celle de Deborah Wamer était plus sympathique, on fait semblant, elle ne voulait pas faire peur. J'avais ces deux pôles. Durant tout le travail, je me disais : si on reste dans le monde symbolique comme Peter Brook - parait-il qu'au lieu de sang, c'était des rubans qui coulaient, esthétique peutêtre maintenant démodée qu'on ne referait sans doute plus - on aurait raté quelque chose: le sang, le cru. Si on part de ma proposition que cette pièce traite de la faillite du symbole en général, de la langue, du rituel, de la culture, et qu'elle se trouve déstabilisée, mêlée, emmêlée dans une mixture imbuvable et immonde avec du corps et du sang humain, alors it ne fallait pas être que dans un camp, celui der symbolique, ou dans un autre, celui du sang. A mon sens, et sans aucun jugement de valeur, ce qu'on m'a dit de la mise en scène de Peter Brook, même si elle était géniale, me parâit fausse parce qu'il n'est resté que dans le symbolique - où est le sang, le cru? et ce qu'on m'a dit de celle de Dubois me paraît faux aussi - d'accord le sang, d'accord le cru, mais où est le cuit, le symbole? Je crois qu'il fallait sans arrêt tenir la barre égale entre l'un et l'autre. C'est pour cela qu'il n'y a pas eu de complaisance 'gore' dans le fait de montrer du cru, du sang, de la violence, personnellement, j'ai horreur de ça. Mais je crois aussi qu'il ne fallait pas rester sage et poétique et faire d'un spectacle qui traite de l'horreur une chose belle, sympathique, symbolique, théâtralement réussie avec de jolies trouvailles. On a essayé d'être sur le fil. Certains ont été déçus : oui, mais il y a un peu de sang - c'est la pièce; d'autre me disaient, il n'y a pas assez de sang. Je pense qu'on a joué la pièce en ne tranchant pas.

JMD : J'ai envie de te citer : «Il ne s'agit pas de mettre en scène une fois de plus la violence. Il s'agit moins de montrer l'abomination, la magie meurtrière, l'abjection, la souillure, que de s'interroger devant ce qui dépasse l'endurance».

DM : $\quad$ Montrer cela n'a strictement aucun intérêt, sinon une certaine complaisance sado-maso ou morbide. 
JMD : Qui devait cependant à l'époque répondre à un goût réél dont on a certainement un équivalent très approximatif aujourd'hui dans les 'hard movies', Massacre à la Tronçonneuse.

DM : A la différence que, à moins d'erreur, Massacre ... ne traite pas du symbolique et de sa faillite. Voilà pourquoi je dis que cette pièce était diffamée. Bien sûr que Shakespeare devait être "malhonnête", c'est-à-dire plaire à un public grâce à ces représentations-là tout en disant des choses bien plus intelligentes que ce qu'on croyait, en leur délivrant de manière souterraine une vraie réflexion sur ce qu'était la violence. Dans ces quelques lignes, il ne s'agit pas de Massacre ..., il s'agit de montrer un spectacle qui s'interroge sur "pourquoi Massacre ...", un en-deça de Massacre ... Malheureusement, on connait ce déploiement d'horreurs de manière fictive ou réélle. Pourquoi à un moment, le livre ne suffit plus, on va tuer. J'ai une anecdote rapide à raconter à propos de l'affaire Rushdie, sans doute fausse parce qu'on me l'a racontée en l'enjolivant pour me plaire, et que je l'enjolive à mon tour de manière légendaire, mais tout ceci est orthodoxe. On m'a dit qu'il y avait eu une émission de télévision où on avait réuni des gens à propos de l'appel au meurtre lancé par l'ayatollah Khomeiny, dont l'inévitable Bernard-Henry Lévy, le Grand Rabin, un représentant de l'ayatollah de l'Ambassade d'Iran. B.-H. Lévy aurait dit : nous les Juifs, on ne condamne pas à mort. Le Grand Rabin aurait dit: je regrette, si, - ce qui a jeté un froid terrible - quand on blasphème sur Dieu ou le Prophète, c'est une transgression de la loi, car on ne vit que sur la loi, la loi doit réagir en condamnant à mort. La différence, c'est que nous, dans l'histoire du peuple juif, on ne l'a jamais fait. L'écrivain Salman Rushdie a fait une entorse symbolique, il est condamné à mort symboliquement - symbole contre symbole, tout va bien. L'ayatollah passe à l'acte, du sang va couler. Il n'y a pas de précédent dans la loi juive parce qu'il est écrit que celui qui blasphème doit le faire trois fois dans les mêmes conditions avant d'être exécuté. Imaginons qu'un jour, sur la place du marché, quelqu'un dise «Dieu est un porc». Attention, il va être condamné à mort, il n'a plus que deux fois. Mais le lendemain quand il le redit, ce n'est pas le même climat, il fait plus frais; il y avait trois oranges sur l'étal, maintenant, il n'y en a plus que deux : on ne trouve jamais les mêmes conditions; donc on n'a jamais tué quelqu'un vraiment, mais il est condamné à mort. Shakespeare dit un peu cela : tant que vous êtes dans le symbolique, tout va bien, 
dès que vous passez à l'acte et que vous faites couler du sang, c'est l'horreur.

\section{QUESTION}

Qu'est-ce qui vous a donné l'idée du personnage aux pigeons?

DM : $\quad$ Ce personnage existe dans la pièce de Shakespeare : c'est le marchand d'oiseaux. C'est le clown, au sens élisabéthain, celui qui vient de l'extérieur pour faire rire, personnage hétérogène de comédie qui arrive au milieu d'une tragédie. Ceci est manifeste dans l'écriture puisque toute la tragédie est en vers sauf les dialogues de ce personnage. Je l'ai trafiqué parce que je n'ai pas pu m'en empêcher : $j$ 'en ai fait des colombes et non pas des perdrix qu'il a braconnées et qu'il vend sur la place du marché.

Ann LECERCLE: Voilà la différence entre la lettre de la loi et l'esprit de la loi. La première étant écrite avec de la chair, la seconde avec de l'encre.

DM : Dans Shakespeare, tout est toujours possible, chaque lecture cohérente est vraie. On peut avoir une lecture mystique, presque juridique, métaphysique, psychanalytique, et chaque fois on trouvera des vérifications. La lecture que j'ai faite, mais il y en aura d'autres, car on n'a pas "brûlé" le livre après l'avoir joué, est celle qui rappelle que le thếtre est le jeu du symbole et du corps, du cru et du cuit.

Jean-Pierre VILLQUIN : Je voulais d'abord féliciter DM pour le plaisir intellectuel et esthétique qu'il nous a donné dans ce spectacle. Ma question, marginale par ailleurs, est la suivante: est-ce le résultat d'un certain parti-pris que d'avoir gommé l'aspect romain de la pièce et d'avoir remplacé les personnages par des personnages sortis de l'Ancien Testament?

DM : $\quad$ Ce n'était pas vraiment l'Ancien Testament, ils étaient habillés à la mode 1930, avec des cravates, des gilets, des chaussures à lacets. Mais je suis très heureux que vous ayez parlé d'Ancien Testament. Il faut presque se transformer en cabaliste pour bien lire Shakespeare. Quand on dit que la pièce se passe à Rome, est-ce simplement un lieu géographique, un lieu dans l'histoire? Est-ce que cela aurait pu se passer à Vérone, Elseneur, Venise? Je crois que Rome veut dire quelque chose, non pas la 
ville qu'on connaît, l'Empire. Qu'est-ce que Rome pour un Elisabéthain? Je suppose que c'est la dernière grande civilisation en date avant le siècle d'Elisabeth, c'est la civilisation et la culture d'où nous somme issus, nous les Elisabéthains, dirait Shakespeare. C'est plutôt une origine, paternelle ou maternelle. Si Shakespeare était rééllement notre contemporain, aurait-il écrit que la pièce se passait à Rome? Je n'en sais rien, ma question est évidemment un peu naïve, mais il aurait peut-être dit que cela se passait au début du temps, comme un Beckett, juste là maintenant. Je me suis demandé ce qu'était pour moi l'origine : l'origine proche est mon papa, l'origine lointaine est l'aube de l'humanité, les premières vraies civilisations, les Hébreux avant même le début de la Bible. Voilà ce que j'ai demandé à la costumère Laurence Forbain: il s'agit donc d'habiller les acteurs comme mon papa et la Bible. Dans ce sens il me semble que c'est "shakespeariennement" romain, et non pas mécaniquement romain. Et où sont les jupettes avec les jambes poilues qui sortent d'habitude, et les colonnes en stuc ... ?

JMD : Cela ne s'est pratiquement jamais fait pour Titus Andronicus, avec le type d'exhibition anatomique que tu décris là.

UNE DAME : Il y a beaucoup de lectures de Shakespeare, dont une lecture historique, non pas en montrant Rome, mais la cruauté à l'époque élisabéthaine, suivant d'autres Elisabéthains comme John Ford, dans un moment d'histoire concret.

DM : Vous voulez dire «Rome n'est pas Rome, mais c'est le siècle d'Elisabeth»? On peut aussi dire «Rome n'est pas Rome, mais c'est de nos jours, et c'est la guerre de 39-45» ... A partir du moment où on dit "Rome n'est pas Rome...», on a intérêt à ne pas s'ancrer. N'importe quel processus tel que celui qui est décrit dans la pièce de Shakespeare est vrai à un certain moment de l'histoire - les années 30 et quelque chose de biblique donnent irrésistiblement à penser à la guerre 39-45; au massacre des Juifs ... Des gens m'ont dit que c'était une allégorie pour mieux montrer l'holocauste nazie, oui ou non, je n'ai jamais dit explicitement que nous allions monter l'holocause nazie grâce à Titus Andronicus. Mais à partir du moment où c'est un cheminement mental, on peut aussi y voir l'époque élisabéthaine si on est historien, malheureusement, $98 \%$ des spectateurs ne connaissent pas cette époque. En tout cas c'est un processus mental travaillant l'histoire vraie, conrète, et non pas le choix illustré de telle époque précise. 
Professeur RICHER : Je voudrais parler des noms des personnages Tamora et Aaron. Shakespeare aurait très bien pu prendre le nom de Tamiris, nom d'une reine ayant existé historiquement, mais il a choisi un nom qui, chose extraordinaire et qui $\mathrm{m}$ 'a beaucoup étonné, est un nom turc qui veut dire "reine de l'enfer". De même le nom de Aaron est d'apparence juive, mais si vous regardez le répertoire des constellations, on remarque qu'Áaron est un des noms de la constellation du Serpentaire, Ophiuchus, qui renvoit au signe du Scorpion. Ce qui me frappe, et rejoint ce qui vient d'être dit, c'est que cette pièce a un caractère expérimental, et que par la suite, Shakespeare n'a cessé d'y puiser. Le plus frappant étant d'ailleurs le trio constitué par Tamora, Aaron et Saturninus, qui préfigure très exactement le trio infernal du Roi Lear, constitué par Regan, Goneril et Cornouaille. On pourrait en dire autant des personnages de victimes dont on n'a pas parlé jusquà présent, ce qui m'étonne, car Shakespeare ne cessera de les reprendre en les modifiant : Lavinia dérive de Lucrèce. Il y a des reprises presque textuelles des poèmes qui sont signalées dans les éditions critiques. Dans l'ensemble, il y a intérêt à orienter les interprétations que l'on peut donner de cette pièce dans le sens symbolique, - le sang, nous savons qu'il en coule suffisamment de nos jours.

Margaret JONES-DAVIES : L'autre jour, je suis allée voir Le Cercle des Poètes disparus, qui comprend cette scène merveilleuse où un professeur fait monter tous ses élèves sur la table un peu pour changer la perspective de leur regard. Cela m'a rappelé mon expérience quand j'ai vu Titus Andronicus : pour la première fois de ma vie, je me suis trouvée étendue par terre en train de regarder une coupole au lieu d'être assise dans un fauteuil. Cela a été un choc extraordinaire. Déjà la position dans laquelle vous nous avez mis dans le thêâtre lui-même est bouleversante littéralement - c'est bien le mot qui vient à l'esprit quand on vous entend et qu'on voit la pièce : tout est un problème de littéralité. Et justement, je suis en train de travailler sur les problèmes de littéralité au Moyen Age dans l'exégèse biblique. Votre mise en scène correspond tout à fait à la manière dont certains exégètes considéraient le texte biblique: il y avait deux possibilités : soit suivre le texte à la lettre et rajouter des petits symboles comme ornements, soit avoir une attitude rationnelle en partant de la lettre, mais en même temps lui faire subir toutes les transformations que 
vous lui faites subir, vous. Cela a été une véritable création à partir de la lettre elle-même. Il faut défendre ce genre de lecture de Shakespeare, puisqu'on a dû vous accuser d'avoir abîmé le texte. J'ai apporté le programme car j'ai été frappée par cette citation de Kantor: «J'attache au texte de la pièce une importance beaucoup plus grande que ceux qui prônent la fidélité au texte, qui le considèrent comme point de départ et qui s'en tiennent là.» Et c'est justement ce qui se passe actuellement au théâtre : on est fidèle au texte, mais qu'est-ce qu'on s'ennuie! Dans votre cas, on n'est pas fidèle au texte de manière piétiste, c'est une fidélité qui introduit l'allégorie, le symbole. C'est vraiment du théâtre comparé à toutes ces pièces trop fidèles et qui finalement ne disent rien.

JMD : Ce serait une bonne conclusion.

Marie-Thérèse JONES-DAVIES : Moi aussi je voudrais remercier Monsieur Mesguich parce que j'ai beaucoup apprécié sa pièce. Je vais peut-être tomber à côté mais cela m'a fait penser à Umberto Eco.

DM : Tout à fait. Ce n'est pas à côté. J'ai beaucoup pensé à Umberto Eco.

Texte transcrit par Isabelle GASTINE-SCHWARTZ. 